\title{
Majority and Plurality Problems
}

\author{
Dániel Gerbner, Gyula O.H. Katona, Dömötör Pálvölgyi and Balázs Patkós
}

June 16, 2018

\begin{abstract}
Given a set of $n$ balls each colored with a color, a ball is said to be majority, $k$ majority, plurality if its color class has size larger than half of the number of balls, has size at least $k$, has size larger than any other color class; respectively. We address the problem of finding the minimum number of queries (a comparison of a pair of balls if they have the same color or not) that is needed to decide whether a majority, $k$-majority or plurality ball exists and if so then show one such ball. We consider both adaptive and non-adaptive strategies and in certain cases, we also address weighted versions of the problems.
\end{abstract}

\section{Introduction}

Two very much investigated problems in combinatorial search theory are the so-called majority and plurality problems. In this context, we are given $n$ balls in an urn, each colored with one color. A majority ball is one such that its color class has size strictly larger than $n / 2$. A plurality ball is one such that its color class is strictly larger than any other color class. The aim is either to decide whether there exists a majority/plurality ball or even to show one (if there exists one). Note that if the number of colors is two, then the majority and the plurality problems coincide. Although there are other models (e.g [6]), in the original settings a query is a pair of balls and the answer to the query tells us whether the two balls have the same color or not. Throughout the paper we consider queries of this sort.

The first results in this area of combinatorial search theory are due to Fisher and Salzberg [7] and Saks and Werman [9]. In [7] it is proved that if the number of possible colors is unknown, then the minimum number of queries in an adaptive search for a majority ball is $\lfloor 3 n / 2\rfloor-2$, while [9] contains the result that if the number of colors is two, then the minimum number of queries needed to find a majority ball is $n-b(n)$, where $b(n)$ is the number of 1 's in the binary representation of $n$. The latter result was later reproved in a simpler way by Alonso, Reingold, and Schott [3] and Wiener [11]. 
The adaptive version of the plurality problem was first considered by Aigner, De Marco, and Montangero in [2], where they showed that for any fixed positive integer $c$, if the number of possible colors is at most $c$, then the minimum number of queries needed in an adaptive search for a plurality ball is of linear order, and the constants depend on $c$. Non-adaptive and other versions of the plurality problem were considered in [1].

Non-adaptive strategies were also studied by Chung, Graham, Mao and Yao [4, 5]. They showed a linear upper bound for the majority problem in case the existence of a majority color is assumed. They mention a quadratic lower bound without this extra assumption. We precisely determine the minimum number of queries needed. They also obtain lower and upper bounds on the plurality problem in the non-adaptive case. We improve those bounds and find the correct asymptotics of the minimum number of queries.

To state our results we introduce some notations. $M_{c}(n)$ denotes the minimum number of queries that is needed to determine if there exists a majority color and if so, then to show one ball of that color and $P_{c}(n)$ denotes the minimum number of queries that is needed to determine if there exists a plurality color and if so, then to show one ball of that color. In both cases the subscript $c$ stands for the number of possible colors. The corresponding non-adaptive parameters are denoted by $M_{c}^{*}(n)$ and $P_{c}^{*}(n)$. A ball is said to be $k$-majority if its color class contains at least $k$ balls. $M_{c}(n, k)$ denotes the minimum number of queries that is needed to determine if there exists a $k$-majority color and if so, then to show one ball of that color and $M_{c}^{*}(n, k)$ denotes the parameter of the non-adaptive variant.

We also consider weighted problems. Let $S=\{w(1), \ldots, w(n)\}$ be a multiset of positive numbers, where $w(i)$ is considered to be the weight of the $i$ th ball. The total weight $w=w(S)$ of the balls is $\sum_{i=1}^{n} w(i)$. The weight $w(T)$ of a subset $T \subseteq[n]$ is $\sum_{i \in T} w(i)$. A color is majority if its color class $C$ satisfies $w(C)>w / 2$ and $k$-majority if $w(C) \geq k$ holds. A color is said to be plurality if the weight $w(C)$ of its color class $C$ is strictly greater than the weights of all the other color classes. The appropriate parameters are denoted by $M_{c}(S), M_{c}(S, k), M_{c}^{*}(S), M_{c}^{*}(S, k)$ and $P_{c}(S), P_{c}^{*}(S)$.

For a set $Q$ of queries we define the query graph $G_{Q}$ to be the graph where the vertices correspond to balls and two vertices are joined by an edge if and only if there exists a query in $Q$ that asks for the comparison of the two corresponding balls.

Throughout the paper log stands for the logarithm of base 2 .

In Section 2 we describe a result of Hayek, Kutin and Melkebeek [8] and some further observations that will serve as tools in our proofs. In Section 3 we characterize the query graphs that solve the non-adaptive $k$-majority problem. As a corollary we obtain the following theorem.

Theorem 1.1. Suppose $n \geq c>2$. Then $M_{c}^{*}(n)=\lceil\lceil n / 2\rceil n / 2\rceil$.

In the rest of Section 3 we consider the weighted adaptive majority problem and obtain 
lower bounds on $M_{2}(S)$ and $M_{2}(n, k)$. Our bounds are always at least as good as the one established by Aigner [1], and in some cases our bounds are better.

Section 4 contains bounds on $P_{c}^{*}(n)$ and $P^{*}(S)$. Our main result concerning the plurality problem is the following theorem.

Theorem 1.2. For any pair of integers $n$ and $c$, the following holds:

$$
\left\lceil\frac{1}{2}\left(n-1-\frac{n-1}{c-1}\right) n\right\rceil \leq P_{c}^{*}(n) \leq \frac{c-2}{2(c-1)} n^{2}+n
$$

\section{Tools}

In this section we introduce a result of Hayes, Kutin and Melkebeek [8] that we will use in Section 3. We also make an easy observation that will serve as a tool in Section 4 .

Let us start with describing the context of the result by Hayes, Kutin and Melkebeek. Let $f$ be a Boolean function of $n$ variables $x_{1}, x_{2}, \ldots, x_{n}$. A parity question is a subset $T \subseteq[n]$ and the answer to this question is $\sum_{i \in T} x_{i}$ modulo 2. Let us define $\mathcal{D}^{\mathcal{P} \mathcal{A R \mathcal { T }} \mathcal{Y}}(f)$ to be the minimum number of parity questions needed to determine the value of $f$.

Lemma 2.1 (Hayes, Kutin and Melkebeek, Lemma 17 in [8]). Let $f$ be a Boolean function on $\{0,1\}^{n}$. If $\mathcal{D}^{\mathcal{P A R} \mathcal{R} \mathcal{T} \mathcal{Y}}(f) \leq d$, then $2^{n-d}$ divides $\left|f^{-1}(1)\right|$.

The proof of the lemma is a straightforward extension of a lower bound by Rivest and Vuillemin [10] for standard decision trees. Note that if $|T|=2$, then the answer to a parity question tells us whether the variables in $T$ have the same value or not. Let $f$ be the function that takes value 1 if there is majority among the values of the variables and 0 if there is no majority. It is easy to see that $f$ is a Boolean function. Using Lemma 2.1 Hayes, Kutin and Melkebeek gave a surprisingly short proof of the lower bound of the result of Saks and Werman [9] which states that for two colors the minimum number of queries needed to find a majority ball is $n-b(n)$, where $b(n)$ is the number of 1's in the binary representation of $n$. Note also that Lemma 2.1 cannot be applied if $n$ is odd. On the other hand it yields a stronger result than that of Saks and Werman in case $n$ is even: Lemma 2.1 gives the same lower bound even if the aim is only to decide whether there is majority and one is allowed to ask parity questions where $|T|=2$ is not required. The proof is to simply apply Lemma 2.1] with $f$ being the majority function and notice that the largest two power that divides $\sum_{i>n / 2}\left(\begin{array}{c}n \\ i\end{array}\right)=2^{n-1}-\left(\begin{array}{c}n \\ n / 2\end{array}\right) / 2$ is $2^{b(n)}$ (this follows e.g. from Kummer's theorem).

Let us finish this section with an easy observation on how complete multipartite graphs can be used in detecting color classes of balls. The next observation will be only used in the non-adaptive case. 
Observation 2.2. If $G_{Q}$ is a complete multipartite graph and $u, v$ are vertices of different classes corresponding to balls of the same color $C$, then all the balls of color $C$ can be identified.

Proof. Any vertex $x$ corresponding to a ball of color $C$ is joined by an edge in $G_{Q}$ to either $u$ or $v$ and therefore there is a query in $Q$ asking whether the ball corresponding to $x$ has the same color as the balls corresponding to $u$ and $v$.

\section{Majority}

In this section we consider majority problems. Let us start with the non-adaptive case. If the number of possible colors is two, then Aigner solved the $k$-majority problem provided $n / 2<k$.

Theorem 3.1 (Aigner, [1]). For $n \geq 3$

$$
M_{2}^{*}(n, k)= \begin{cases}n-1 & \text { if } n<2 k-1 \\ n-2 & \text { if } n=2 k-1 .\end{cases}
$$

Let us continue with the more general, weighted $k$-majority model. The next theorem characterizes the query graphs that solve the weighted $k$-majority problem provided some extra assumptions are satisfied. For simplicity, we will assume that the vertex set of the query graph is $[n]$. Given a multiset $S=\left\{w_{1}, w_{2}, \ldots, w_{n}\right\}$ of weights let $\mathcal{F}=\{F \subset[n]: w(F) \geq k\}$ be the family of the $k$-majority sets. Let $\mathcal{F}_{0}$ denote the subfamily of minimal sets in $\mathcal{F}$.

Theorem 3.2. Suppose there are no 1-element sets in $\mathcal{F}$. Then

(i) If each member of $\mathcal{F}_{0}$ induces a connected subgraph of the query graph $G_{Q}$, then $G_{Q}$ solves the weighted $k$-majority problem.

(ii) If $2 w([n])<(k+1)(c+1)-2, c>2$ and $G_{Q}$ solves the weighted $k$-majority problem, then each member of $\mathcal{F}_{0}$ induces a connected subgraph of $G_{Q}$.

(iii) Considering the non-weighted version, suppose $k$ is an integer. If $n \leq c k-k-c+2$, $c>2$ and $G_{Q}$ solves the $k$-majority problem, then each member of $\mathcal{F}$, i.e. any set with at least $k$ elements, induces a connected subgraph of $G_{Q}$.

Proof. (i) If the induced subgraph on $F$ is connected, then one can easily check if $F$ is monochromatic. By the assumption, a minimal $k$-majority set of each candidate for $k$ majority can be checked.

(ii) Suppose $F_{0} \in \mathcal{F}_{0}$ is not connected. Suppose the answers are according to a coloring satisfying the following: one component of $F_{0}$ is blue, all the others are red, and the remaining $c-2$ colors are used on the other vertices in such a way that none of those colors are in 
$k$-majority. If such a coloring exists, there is no $k$-majority, but it cannot be distinguished from the case when every vertex in $F_{0}$ is blue (what would make blue a majority color).

To prove that such a coloring exists, we have to show that there is a partition of $[n] \backslash F_{0}$ into $c-2$ color classes $A_{1}, A_{2}, \ldots, A_{c-2}$, such that $w\left(A_{i}\right) \leq k-1$ for all $i$. The conditions imply $w\left([n] \backslash F_{0}\right)<(k+1)(c-1) / 2$. Notice that this is a Bin Packing problem; we have to prove that certain items with weight sum $<(k+1)(c-1) / 2$ fit into $c-2$ bins. Indeed, it is well-known (and easy to see) that if $c-1(\geq 2)$ bins are required then the sum has to be at least $(c-2)\lceil(k+1) / 2\rceil+\lfloor(k+1) / 2\rfloor \geq(k+1)(c-1) / 2$, we are done.

(iii) The proof goes similarly to the previous case. Suppose $F \in \mathcal{F}$ is not connected. By removing elements from $F$ one by one such that we make sure that at least two components of $F$ do not get totally removed, we obtain a subset $F^{\prime}$ of $F$ such that $F^{\prime} \in \mathcal{F}_{0}$ and $F^{\prime}$ is not connected. By the assumption on $n$, we know that $\left|[n] \backslash F^{\prime}\right| \leq(c-2)(k-1)$. Let us partition $[n] \backslash F^{\prime}$ into $c-2$ color classes each containing at most $k-1$ elements. If one component of $F_{0}$ is colored blue and all other components are colored red, then there is no $k$-majority. But this coloring cannot be distinguished from the case when every vertex in $F_{0}$ is blue (what would make blue a majority color).

Corollary 3.3. Suppose $c>2, n>k>n / 2$ and $n>1$. Then a query graph $G_{Q}$ solves the $k$-majority problem if and only if $G_{Q}$ is $(n-k+1)$-connected.

Proof. Suppose first that $G_{Q}$ is $(n-k+1)$-connected and let $F \in \mathcal{F}$, i.e. $|F| \geq k$ and thus $|[n] \backslash F| \leq n-k$. By definition $G_{Q}$ stays connected after removing the vertices in $[n] \backslash F$ and thus by Theorem 3.2 (i) $G_{Q}$ solves $k$-majority.

Suppose now $G_{Q}$ solves the $k$-majority problem. A simple calculation shows that the assumption of Theorem 3.2 (iii) holds, thus all $k$-subsets of $[n]$ induce a connected subgraph of $G_{Q}$, thus $G_{Q}$ is $(n-k+1)$-connected.

Proof of Theorem 1.1. Clearly, the majority problem is the $k$-majority problem with $k=$ $\lfloor n / 2\rfloor+1$. Thus by Corollary 3.3 any query graph $G_{Q}$ that solves the majority problem must be $\lceil n / 2\rceil$-connected and it is well known that the minimum number of edges that $\lceil n / 2\rceil$-connected graphs can have is $\lceil\lceil n / 2\rceil n / 2\rceil$.

Let us note here that the upper bound of Theorem 1.1 holds also in the weighted case, but such general lower bound cannot be found without extra assumptions on the multiset $S$ of weights. Indeed, if $w_{1}>\sum_{i=2}^{n} w_{i}$, holds, then without any query one knows that the ball with weight $w_{1}$ is a majority ball.

Let us now turn our attention to adaptive majority problems. We will only address problems where the number of colors is two. Apart from the results by Fisher and Salzberg 
[7] and Saks and Werman 9] mentioned already in the Introduction, we are aware of one more major result. If $\mu(n)$ denotes the largest integer $l$ such that $2^{l}$ divides $n$, then Aigner's result can be formulated in the following way:

Theorem 3.4. For any pair of integers $n \geq k>n / 2$, the inequality $M_{2}(n, k) \geq n-1-$ $\mu\left(\left(\begin{array}{l}n-1 \\ k-1\end{array}\right)\right)$ holds.

Note that this result is a generalization of the theorem of Saks and Werman as if $n$ is even, then for $k=n / 2+1$ we have $\mu\left(\left(\begin{array}{c}n-1 \\ n / 2\end{array}\right)\right)=b(n)-1$, where $b(n)$ is the number of 1's in the binary representation of $n$.

We establish two lower bounds on $M_{2}(n, k)$, which easily follow from Lemma 2.1 .

Proposition 3.5. Let $k>n / 2$. Then

$$
M_{2}(n, k) \geq n-1-\mu\left(\sum_{i=k}^{n}\left(\begin{array}{l}
n \\
i
\end{array}\right)\right) .
$$

Proof. Let $f:\{0,1\}^{n} \rightarrow\{0,1\}$ be the function defined by $f(\mathbf{x})=1$ if and only if $\sum_{i=1}^{n} x_{i} \geq k$ or $\sum_{i=1}^{n} x_{i} \leq n-k$, i.e., there is a $k$-majority color. This is clearly a Boolean function and $\left|f^{-1}(1)\right|=2 \sum_{i=k}^{n}\left(\begin{array}{c}n \\ i\end{array}\right)$. The statement now follows from Lemma 2.1.

We can compare this bound to Aigner's lower bound. The example in [1] which shows that Aigner's bound is not optimal is $n=9$ and $k=6$, where his result only gives 5 , while the truth is 7 . Our bound establishes the correct value 7 . On the other hand if $n=10$ and $k=8$, our bound yields only 6 , Aigner's result gives 7 , while the truth is 8 .

Proposition 3.6. Let $k>n / 2$. Then

(i) Let us fix an arbitrary ball. The number $\operatorname{Fix}_{2}(n, k)$ of questions needed to determine if the fixed ball is a $k$-majority ball is at least $n-1-\mu\left(\sum_{i=k}^{n}\left(\begin{array}{c}n-1 \\ i-1\end{array}\right)\right)$.

(ii) $M_{2}(n, k) \geq n-2-\mu\left(\sum_{i=k}^{n}\left(\begin{array}{c}n-1 \\ i-1\end{array}\right)\right)$.

Proof. Lemma 2.1 implies (i) as before, and (ii) follows as $\operatorname{Fix}_{2}(n, k) \leq M_{2}(n, k)+1$. Indeed, if we solve the $k$-majority problem and the answer is that there is no $k$-majority color, then the fixed ball cannot be a $k$-majority ball. If the answer is a $k$-majority ball, then after comparing this to our fixed ball we can decide whether the fixed ball is also $k$-majority or not.

Comparing Theorem 3.4. Proposition 3.5 and Proposition 3.6 (ii), one observes that any two statements imply the third one. First note that $n-2-\mu\left(\sum_{i=k}^{n}\left(\begin{array}{c}n-1 \\ i-1\end{array}\right)\right)$ can be written as $n-1-\mu\left(2 \sum_{i=k}^{n}\left(\begin{array}{c}n-1 \\ i-1\end{array}\right)\right)$ and also the identity $2 \sum_{i=k}^{n}\left(\begin{array}{c}n-1 \\ i-1\end{array}\right)=\left(\begin{array}{c}n-1 \\ k-1\end{array}\right)+\sum_{i=k}^{n}\left(\begin{array}{c}n \\ i\end{array}\right)$ holds. Finally note that for any integers $\alpha, \beta, \gamma$ with $\alpha+\beta=\gamma$ there is no unique minimum of $\mu(\alpha), \mu(\beta)$ and $\mu(\gamma)$. This means that we have also obtained a new proof of Theorem 3.4 . 
Let us now consider the weighted (adaptive) majority problem with two colors. Suppose there are $p \neq 0$ ways to partition the multiset $S$ into two parts of equal weight. Then

Proposition 3.7. (i) At least $n-1-\mu(p)$ questions are needed.

(ii) In case $p$ is even, $n-2$ questions are enough

Proof. Let $f:\{0,1\}^{n} \rightarrow\{0,1\}$ be the function defined by $f(\mathbf{x})=1$ if and only if $\sum_{i: x_{i}=0} w(i)=$ $\sum_{i: x_{i}=1} w(i)$. Again, (i) follows from Lemma 2.1.

For (ii), note that $n$ has to be at least 3 (since zero weights are not allowed). Now we claim that from any three elements there are two that are in different equipartitions an even number of times (and hence also in the same partition an even number of times, so comparing them will keep the property that $p$ is even). The proof is the following. For each partition add an edge between the elements that are in different parts. Each partition gives zero or two edges between our three elements. Thus two of them will be connected with an even number of edges, we are done.

Note that this means $M_{2}(S)=n-1$ iff $\mu(p)=0$. If $\mu(p)=1$, then $M_{2}(S)=$ $n-2$, but the opposite direction is not true, as shown by the following example: $S=$ $\{1,10,11,100,101,110,111\}$. Here $M_{2}(S)=5$ even though $p=4$.

Another possible assumption about the multiset $S$ of weights is that "every element matters", i.e. for every $s \in S$ there exists a coloring of $S \backslash\{s\}$ with two colors (red and blue) such that the majority color is different if we extend this coloring by giving $s$ color red or blue. We say that a multiset $S$ of weights is non-slavery if the above condition is satisfied.

Proposition 3.8. For every non-slavery multiset $S$ of weights the inequality $M_{2}(S) \geq\lfloor n / 2\rfloor$ holds.

Proof. Let $s \in S$ be the element with the smallest weight. There is a partition $S \backslash\{s\}=A \cup B$ such that both $w(A \cup\{s\}) \geq w(V) / 2$ and $w(B \cup\{s\}) \geq w(V) / 2$. Suppose $|A| \geq|B|$. The Adversary can declare all balls corresponding to weights in $B$ to be colored blue and apply the following strategy: for any query $(a, b)$ with $a \in A \cup\{s\}, b \in B$ the answer is DIFFERENT COLOR and for any other query the answer is SAME COLOR. As $s$ is the smallest weight, then by the non-slavery property of $S$ we know that the answer to the majority problem is different if all balls corresponding to weights in $A \cup\{s\}$ are colored red or if there is at least one ball colored blue among those balls. But no matter which set $Q$ of $l$ queries are made with $l<\lfloor n / 2\rfloor$, there will be at least one component $C$ in $G_{Q}$ that lies totally within $A \cup\{s\}$. Now the following two colorings are admissible to $Q$ and give different answers to the majority problem:

1. all balls corresponding to weights in $A \cup\{s\}$ are colored red 
2. balls corresponding to weights in $C$ are colored blue and to those in $(A \cup\{s\}) \backslash C$ are colored red.

\section{Plurality}

As we pointed out in the Introduction, the problem of finding a plurality ball is the same as the problem of finding a majority ball if the number of possible colors is two. In this section we consider the case $c \geq 3$ and determine $P_{c}^{*}(n)$ asymptotically. Our main goal is to prove Theorem 1.2 and then to obtain an upper bound on $P_{c}^{*}(S)$ in the weighted case for general weight set $S$.

The lower bound of Theorem 1.2 immediately follows from the following lemma.

Lemma 4.1. If $Q$ is a set of queries that solve the problem, then the minimum degree in $G_{Q}$ is larger than $n-1-\left\lceil\frac{n-1}{c-1}\right\rceil$. Furthermore, if $n-1 \equiv 1 \bmod c-1$, then the minimum degree in $G_{Q}$ is larger than $n-1-\left\lfloor\frac{n-1}{c-1}\right\rfloor$.

Proof of Lemma. We consider the two cases separately. First, suppose that $n-1 \not \equiv 1 \bmod$ $c-1$ and the degree of a vertex $x$ is at most $n-1-\left\lceil\frac{n-1}{c-1}\right\rceil$. Then one can partition $V\left(G_{Q}\right) \backslash\{x\}$ into $c-1$ sets whose size differ by at most 1 (we call such a partition equipartition), and $V \backslash(N(x) \cup\{x\})$ contains $T_{1}$, one of the largest sets. If the Adversary answers the queries in the following way, we will not be able to tell whether there is a plurality color or not: for any $u, v \neq x$ the answer is SAME COLOR if and only if $u, v$ belong to the same set of the partition and the answer is DIFFERENT COLOR for any query $(u, x)$. In this way, if $x$ has the same color as $T_{1}$, a subset of its non-neighbors, then this color is plurality, while if $x$ has color different from any other vertex, then there is no plurality.

Let us consider the case $n-1 \equiv 1 \bmod c-1$. Then in a $(c-1)$-equipartition of $V\left(G_{Q}\right) \backslash\{x\}$ there is one set $T_{1}$ which is one larger than all other sets. Then consider such a partition of $V\left(G_{Q}\right) \backslash\{x\}$ where $V \backslash(N(x) \cup\{x\})$ contains a small set $T$ of the partition. Let the Adversary answer the queries as above: for any $u, v \neq x$ the answer is SAME COLOR if and only if $u, v$ belong to the same set of the partition and the answer is DIFFERENT COLOR for any query $(u, x)$. Then we cannot answer the problem even if we have the extra information that balls belonging to the same partite set are colored with the same color. Indeed, if $x$ has the same color as all balls in $T$, then there is no plurality: $T_{1}$ and $T \cup\{x\}$ have the same size. Or if $x$ is colored with a color that is different from the color of all other balls, then any ball from $T_{1}$ is a plurality ball.

Proof of the upper bound in Theorem 1.2. It is enough to show a graph $G_{c, n}$ with $\frac{c-2}{2(c-1)} n^{2}+n$ edges such that no matter what colors the balls have, we are able to solve the problem after 
receiving the answers to queries corresponding to edges of $G_{c, n}$. Let $G_{c, n}$ be the $(c-1)$-partite Turán graph on $n$ vertices with a spanning cycle added to each partite set $V_{1}, \ldots, V_{c-1}$.

First, let us observe that if for some $x \in V_{i}, y \in V_{j}$ with $i \neq j$ the answer to the query $(x, y)$ is SAME COLOR, then after receiving answers to all queries we are able to determine the whole color class of $x$ and $y$. Indeed, as $x$ and $y$ belong to different partite sets, for any $v \in V$ at least one of the queries $(x, V),(y, v)$ is asked and therefore we receive a SAME COLOR answer if $v$ belongs to the color class of $x$ and $y$ and a DIFFERENT COLOR answer is $v$ is of different color.

Let $k$ denote the number of color classes $C_{1}, \ldots, C_{k}$ that intersect at least two of the $V_{i}$ 's and let $l$ denote the number of partite sets that are not contained in $\cup_{i=1}^{k} C_{i}$. Any of the remaining $c-k$ color classes is contained in one of the partite sets not covered by $\cup_{i=1}^{k} C_{i}$, thus by the pigeonhole principle $l \leq c-k$. Moreover, if $l=c-k$, then to cover all partite sets, all $c-k$ colors need to be used in different partite sets and thus they should be of the form $V_{j} \backslash \cup_{i=1}^{k} C_{i}$. As we are able to determine all color classes, the proof is finished in this case.

From now on we assume $l \leq c-k-1$. Let us suppose first that $k=0$. Then as all color classes are included in one of the partite sets we have that all but at most one partite sets form one color class each and the last partite set is the union of at most two color classes which we can identify due to the additional spanning cycle.

Thus we may suppose that $l \leq c-k-1$ and $k \geq 1$ hold. This means that the number of already covered partite sets is $c-1-l \geq k$, thus at least one of $C_{1}, \ldots, C_{k}$ has size at least $\left\lfloor\frac{n}{c-1}\right\rfloor$. Therefore it is enough to prove that we are able to identify all other color classes that have size at least $\left\lfloor\frac{n}{c-1}\right\rfloor$. As all remaining color classes are contained in one of the $V_{i}$ 's, their size is at most $\left\lceil\frac{n}{c-1}\right\rceil$. Thus if a $V_{j}$ contains at least two points from $\cup_{i=1}^{k} C_{i}$, then it cannot contain a color class of size at least $\left\lfloor\frac{n}{c-1}\right\rfloor$. On the other hand if a $V_{j}$ contains at most one point of $\cup_{i=1}^{k} C_{i}$, then, due to the spanning cycle, we are able to tell whether it contains a color class of size $\left\lceil\frac{n}{c-1}\right\rceil$ or $\left\lfloor\frac{n}{c-1}\right\rfloor$.

One can improve the upper bound of Theorem 1.2 for $c=3$. It is not hard to show that the following constructions for $G_{Q}$ correspond to query sets that solve the problem: if $n=2 k$, then consider the complete bipartite graph $K_{k, k}$ on the partite sets $\left\{u_{1}, u_{2}, \ldots, u_{k}\right\}$, $\left\{v_{1}, v_{2}, \ldots, v_{k}\right\}$. Add the edges of the paths $\left(u_{1} u_{2} \ldots u_{k}\right)$ and $\left(v_{1} v_{2} . . v_{k}\right)$ and remove the edges of the matching $\left\{\left(u_{i}, v_{i}\right): i=2, \ldots, k-1\right\}$. While if $n=2 k+1$, then consider the complete bipartite graph $K_{k+1, k}$ on the partite sets $\left\{u_{1}, u_{2}, \ldots, u_{k}, u_{k+1}\right\},\left\{v_{1}, v_{2}, \ldots, v_{k}\right\}$. Add the edges of the path $\left(u_{1} u_{2} \ldots u_{k} u_{k+1}\right)$. These observations yield to the following theorem.

Theorem 4.2. (i) $P_{3}^{*}(2 k)=k(k+1)$, (ii) $\frac{1}{2}(k+1)(2 k+1) \leq P_{3}^{*}(2 k+1) \leq \frac{1}{2}(k+1)(2 k+1)+k-1$.

To obtain the lower bound in (i), one has to apply the $n-1 \equiv 1 \bmod c-1$ case of Lemma 4.1. Details are left to the reader. 
Finally, we turn our attention to the non-adaptive weighted plurality problem, i.e. determining $P_{c}^{*}(S)$ for a multiset $S$ of weights. Theorem 1.2 shows that in general we cannot hope for anything better than the number of edges of the balanced complete $(c-1)$-partite graph on $n$ vertices. Our last theorem states that for any multiset of weights the number of edges of the balanced complete $c$-partite graph on $n$ vertices and a linear number of additional queries can solve the problem.

Theorem 4.3. For any multiset $S$ of $n$ weights the inequality $P_{c}^{*}(S) \leq \frac{c-1}{2 c} n^{2}+n-c$ holds.

Proof. Let us partition $S$ into $c$ classes $A_{1}, \ldots, A_{c}$ with $w\left(A_{1}\right) \geq w\left(A_{2}\right) \geq \cdots \geq w\left(A_{c}\right)$ such that $w\left(A_{i}\right)-w(v) \leq w\left(A_{c}\right)$ for any $i \leq c$ and any $v \in A_{i}$. It is easy to see that such a partition exists. Indeed, starting from any partition if $v$ and $A_{i}$ violates this property, we can remove $v$ from $A_{i}$ and add it to $A_{c}$. Let $B$ denote the set of all pairs $\left(v, A_{i}\right)$ such that $v \in A_{i}$ and $w\left(A_{i}\right)-w(v)>w\left(A_{c}\right)$. Put $O=O\left(A_{1}, A_{2}, \ldots, A_{c}\right)=\sum_{\left(v, A_{i}\right) \in B} w\left(A_{i}\right)-w(v)-w\left(A_{c}\right)$. Note that after removing $v$ from $A_{i}$ and adding it to $A_{c}$ the value of $O$ strictly decreases, hence this process must stop after a certain time and the resulting partition satisfies the required property. Note that $w\left(A_{i}\right)-w(v) \leq w\left(A_{c}\right) \leq w(S) / c$, and the sum of the weights in a plurality color must be larger than $w(S) / c$, the average weight of color classes. It implies that a plurality color (in fact any color with weight larger than $w(S) / c$ ) either contains vertices from more than one class, or is equal to $A_{i}$ for some $i$.

Let $G_{0}$ be the complete $c$-partite graph with parts $A_{1}, \ldots, A_{c}$, and add a spanning tree into every part. Let $G$ be the resulting graph. It is well-known that $G_{0}$ cannot have more edges than the $c$-partite Turán graph on $n$ vertices, and the spanning trees add $n-c$ additional edges. We claim that this graph solves the weighted plurality problem. If there is a color with weight larger than $w(S) / c$, then it is either a class $A_{i}$, and in that case the spanning tree shows that it is monocolored, or it contains vertices from at least two parts, and then by Observation 2.2 we can identify all its vertices. Hence all the colors with weight larger than $w(S) / c$ are completely identified, all one has to do is to check if the largest weight appears only once or more.

\section{Acknowledgement.}

We would like to thank Balázs Keszegh and Gábor Wiener for fruitful discussions.

\section{References}

[1] M. Aigner, Variants of the majority problem, Discrete Applied Mathematics 137 (2004) 3-25. 
[2] M. Aigner, G. De Marco, M. Montangerob, The plurality problem with three colors and more, Theoretical Computer Science 337 (2005) 319-330.

[3] L. Alonso, E. Reingold, R. Schott, Determining the majority, Inform. Process. Lett. 47 (1993) 253-255.

[4] F. Chung, R. Graham, J. Mao, A. Yao, Finding Favorites, Electronic Colloquium on Computational Complexity, Report No. 78 (2003).

[5] F. Chung, R. Graham, J. Mao, A. Yao, Oblivious and Adaptive Strategies for the Majority and Plurality Problems, Computing and combinatorics, Lecture Notes in Computer Science 3595, Springer, Berlin (2005), 329-338.

[6] G. De Marco, E. Kranakis, G. Wiener, Computing Majority with Triple Queries, Proceedings of COCOON 2011, Lecture Notes inComputer Science 6842, 604-611.

[7] M.J. Fisher, S.L. Salzberg, Finding a Majority Among $n$ Votes, J. Algorithms 3 (1982) 375-379.

[8] T.P. Hayes, S. Kutin, D. van Melkebeek, On the Quantum Black-Box Complexity of Majority, Algorithmica 34 (2002) 480-501.

[9] M. E. Saks, M. Werman, On computing majority by comparisons, Combinatorica 11 (1991), 383-387.

[10] R. Rivest, J. Vuillemin, On recognizing graph properties from adjacency matrices, Theoret. Comput. Sci. 3 (1976) 371-384.

[11] G. Wiener, Search for a majority element, J. Stat. Plann. Inf. 100 (2002) 313-318. 